\title{
A Combinatorial Optimization Solution for Activity Prioritizing Problem
}

\author{
Mohammad Ali Hatefi ${ }^{*}$, Seyyed Abdollah Razavi \\ ${ }^{1}$ Department of Energy Economics \& Management, Petroleum University of Technology (PUT); Postal address: Sattarkhan Ave., Khosrow \\ Jonoubi St., Tehran, Iran; Telephone no.: 098-21-44222483, Mobile no.: 09125403684; E-mail: Hatefi@put.ac.ir \\ ${ }^{2}$ Department of Energy Economics \& Management, Petroleum University of Technology (PUT); Postal address: Sattarkhan Ave., Khosrow \\ Jonoubi St., Tehran, Iran; Telephone no.: 098-21-44222483, Mobile no.: 09122490460; E-mail: Srazavi@put.ac.ir
}

\begin{abstract}
This paper discusses a special situation in project management in which an analyst wants to prioritize several independent activities to handle all them one after another, in such a way that there are no precedence relationships over the activities. As a novel idea, in this research, the notion is that the structure of prioritized activities is a linear arrangement, and therefore it can be taken into account as a combinatorial optimization problem. The paper formulates a mathematical model, and employes it in two real cases from oil and gas industry. In addition, the paper develops a row generation procedure to solve large scale problems, and reports the computational results for the problem instances of size up to 300 activities. The results demonstrate the applicability and efficiency of the proposed methodology.
\end{abstract}

Keywords: Activity Prioritizing Problem; Project scheduling; Mathematical programming; Row generation; Combinatorial optimization; Branch-and-cut, Oil and gas industry.

\section{Introduction}

As Gerardi [1] states in his book, project managers generally list several explanations as to why they love project management, such as they get to learn new things, work with new people, gain new skills, and are on the leading edge of innovation. In line with this view, this paper concentrates on an interesting challenge in project planning named Activity Prioritizing Problem (APP) which is a branch of project scheduling problem. Project scheduling has attracted considerable attention because of its critical role in project resource management [2, 3]. Consider a situation in which there are project activities with no precedence relationships over them. For instance, after scheduling a lot of project activities, a subset of them (with no inter-related dependencies) should be done in a limited period of time. In this situation, the problem simply is to assign priorities to such activities. It is clear that this challenge is not affected by duration of activities. Instead, several factors likely influence the priorities, such as project' owner view, program and project manager' ideas, risk factors [4], etc. In this regard, the role of project manager and project experts have been addressed as fundamental dimensions for the project success [5], although this fact has been ignores by the literature [6, 7]. On the other hand, Greek and Pullin [8] argue that many project managers do not focus on the criticality and urgency of project activities. Whereas a major concern in project management is the determination of a priority ranking for all activities that are candidates for assignment [9].

With the APP, there are many descriptive solutions and a few analytical models in the relevant literature. Among various descriptive guidelines (6-step process, Eisenhower matrix, Brian Tracy's method, ABCDE method, Bubble sort technique, Moscow method, Pareto principle, Zen habit, Rag rating system, Scrum prioritization, 13-9 method, and so on) let have little explanations for only two methods. According to the Zen habit method 
[10], as a really simple prioritization guideline, each time the manager writes down one to three most important activities that he/she wants to complete on a limited period of time, and get on with completing them. The Eisenhower matrix, popularized by Covey [11], works by making segregate activities into four quadrants based on urgency and importance: urgent and important, important but not urgent, urgent but not important, and neither urgent nor important.

Despite the importance of the weighting and prioritizing project activities, analytical models have been rarely addressed in the related literature [7, 12]. In addition, most of the analytical studies use Multiple Attribute Decision Making (MADM) techniques to get a rank or weight vector of activities. Chang and Ibbs [9] introduced an expert system that uses possibility theory and the generalized modus ponens logic inference rule to prioritize activities in building construction projects. Alencar et al. [13] employed ELECTRE III technique to prioritize activities in a real-world construction project. Jha and Misra [14] made a survey to identify major factors in classifying and ranking of construction coordination activities. An analytical effort was performed by Mota et al. [15] who presented a model for supporting project managers to focus on the main activities of a project network using a MADM technique, taking into account several, often contradictory, points of view. That model aims to assign project activities into three classes of managerial practice. In an article by Baykasoglu et al. [12], a practical fuzzy rating and ranking technique was proposed to prioritize project activities with fuzzy attributes. They considered prioritization of activities instead of their classification. In addition, their technique was in some aspects similar to Mota et al.'s model [15], with substantial differences. Vanhoucke [16] introduced a scheme to measure the activities sensitivity and network topological information to monitor the project time performance. Mota and de Almeida [17] proposed an ELECTRE-based model to help project managers taking decisions about the key activities of project. Golpira [18] made a comparison between conventional MADM and Fuzzy MADM to determine activities weights and priorities using Analytical Hierarchy Process (AHP) and Technique for Order of Preference by Similarity to Ideal Solution (TOPSIS) methods. Hadad et al. [19] prioritized the project activities attributes using an MADM model. These attributes include activities expected time, activities standard time deviation, activities expected cost, activities standard cost deviation and the activities time and cost obtained through simulation. Hadad [20] proposed a technique to prioritize project activities using AHP and Data Envelopment Analysis (DEA) as the research tools to select the proper influencing factors. Kalayathankal et al. [21] applied a fuzzy technique to prioritize activities in a software development project with the analogous activities in the electrical substation construction studied by Mota et al. [15]. They claimed brought out better project output in terms of finance, personnel, equipment, space, time etc. Berjis et al. [7] proposed an approach to determine the weights of project activities using DEA. They identified the parameters affecting the importance of activities through a review of the related literature and experts' opinions. The parameters included activity duration, activity cost, activity importance, activity difficulty, safety, communication rate, intellectual effort, physical effort, unfavourable work conditions and work related hazards.

In the current article, a novel optimization solution is proposed for a special situation of the APP. The idea in the proposed approach is completely different from previous works. The organization of the paper is as follows. Section 2 points out the problem characterization and related definitions. Section 3 develops a mathematical 
model to solve the problem. Section 4 includes two real cases studied from oil and gas industry. In section 5, the paper discusses how to deal with large scale problems followed by experimental analysis in section 6. Finally, section 7 summarizes the results achieved, and provides future efforts possibilities.

\section{Characterization of the problem}

Herein, a special situation of the APP is introduced. This situation is an interesting issue which the authors encountered it in real projects. Indeed, real cases (described in section 4) were the root of the idea of the problem discussed in this paper. As a result, the need for the proposed model (described in section 3) originates from real-world projects, and thus can be employed in actual challenges.

Suppose a situation in which there are a variety of project activities and the aim is to prioritize activities to handle all them one after another. Indeed, all activities need a special unique resource which is available only for one activity in any point of the time. Taking this fact into account, we want to sequentially perform activities from the most important to the least important. Thus an arrangement structure of activities needs to be formed. That special resource may be a unique montage platform, a unique consultant, a unique working front, etc. The solution is influenced by some different "adjacency factors". An adjacency factor indicates a reason whereby some activities need to be assigned the locations in the arrangement structure close to each other. Obviously, any linear permutation including all activities is a valid solution for the problem. Thus, the idea behind the current paper arises from this point of view that in the prioritizing challenge explained above is a linear arrangement actually; and therefore it is a combinatorial optimization problem in which activities should be arranged from the most important to the least important.

Let us define the problem in a general form. The problem has two groups of elements: "activities" and "adjacency factors" (henceforth referred to as factors). There are $m$ activities $T_{1}, T_{2}, \ldots, T_{m}$ to be prioritized and $n$ factors having positive weights $\gamma_{1}, \gamma_{2}, \ldots, \gamma_{n}$. In view of an assumed factor, the relevant activities should be placed one after another in the overall arrangement structure. The activities respecting $j$ th factor are shown by subset $f_{j} \subset\left\{T_{1}, \ldots, T_{m}\right\}$. An assumed factor may be proposed by any of $o$ experts $(k=1,2, \ldots, o)$. Additionally, each expert may suggest various factors. The factors are actually derived from similarity of the complexity of activities, relationships among activities, equality in urgency of activities, etc. The weight of a given factor is calculated as Equation (1) which is weighted geometric mean stressed by many authors to aggregate the individual weights [22-25]. In this equation $w_{j k}$ is the weight of $j t h$ factor preferred by $k t h$ expert. This individual weigh is a number between 1 and 10. If an expert has no idea about a factor, the respected items should be dropped out from Equation (1). The power $u_{k}$, a number between 0 and 1, indicates the importance of $k t h$ expert's comments. This power depends on the expert's skills, experiences, abilities, or his/her area of educations.

$\gamma_{j}=\left(\prod_{k=1}^{o} w_{j k}^{u_{k}}\right)^{1 / \sum_{k=1}^{o} u_{k}}, \quad j=1, \ldots, m$ 
In view of $j$ th factor, the best case is comprised of a contiguous chain of all the activities in $f_{j}$. In such a case, complete weight $\gamma_{j}$ will be added to the objective value of the problem mathematical model; otherwise, a proportion of $\gamma_{j}$ would be met. Taking this into consideration, the problem is to arrange activities so as to maximize the sum of proportions of factors weights. The value of each proportion depends on the adjacency of relevant activities in the overall arrangement structure.

Truly, the introduced challenge falls into combinatorial optimization problem. Although a wide range of models and algorithms exists to deal with famous graph-theoretic and combinatorial optimization problems, the introduced issue is not like any well-known problems. In particular, it is completely different from Cell Formation Problem [26, 27], Job-Shop Scheduling Problem [28], Project Scheduling Problem [2], Single Row Facility Layout Problem [29], Linear Ordering Problem [30], Minimum Linear Arrangement Problem [31, 32], Adjacency Problem [33], MADM problem [22, 34], and Linear Assignment Problem [35].

\section{Modeling the problem}

First, let us define:

- $\quad T_{i}$ refers ith activity $(i=1, \ldots m)$.

- $f_{j}$ stands for the set of activities respecting $j$ th factor $(j=1, \ldots n)$.

- $\quad \gamma_{j}$ indicates the weight of $j$ th factor $(j=1, \ldots n)$.

- $\theta_{p q}(p<q)$, a binary variable, equals one if activity $T_{p}$ is placed exactly adjoining activity $T_{q}$.

- $s()$ shows size of a set.

Now, we write formulation of the problem as Model (2)-(5).

$\operatorname{Max} \sum_{j=1}^{n}\left(\frac{\sum_{T_{p} \& T_{q} \in f_{j} \mid p<q} \theta_{p q}}{s\left(f_{j}\right)-1}\right) \gamma_{j}$

S.t.

$\sum_{q=1, \ldots, m \mid i<q} \theta_{i q}+\sum_{p=1, \ldots, m \mid p<i} \theta_{p i} \leq 2, \quad i=1, \ldots, m$

$\sum_{T_{p} \& T_{q} \in B \mid p<q} \theta_{p q} \leq s(B)-1, \quad B\left\{T_{1}, \ldots, T_{m}\right\}, 2<s(B)$

$\forall \theta_{p q} \in(0,1)$

For the sake of better understanding, an explicit formulation for a small problem with corresponding LINGO code was provided in Appendix. 
The rule to formulate Function (2) is "If two activities of $f_{j}$ is placed right beside each other in the overall string of activities, then a " 1 " is added to objective function coefficient of $\gamma_{j}$ ". Let us consider some cases. As an instance, suppose that there are 4 activities and 3 factors, and we want to determine the objective function coefficients of $\gamma_{1}, \gamma_{2}$ and $\gamma_{3}$, for given string $T_{4} T_{1} T_{3} T_{2}$. The set of activities respecting the factors are $f_{1}=\left\{T_{1}, T_{3}, T_{4}\right\}, f_{2}=\left\{T_{2}, T_{4}\right\}$, and $f_{3}=\left\{T_{1}, T_{2}, T_{4}\right\}$. For a given factor $f_{j}$, at the best case, in which each activities of $f_{j}$ has at least one adjacent activity from $f_{j}, \sum_{T_{p} \& T_{q} \in f_{j} \mid p<q} \theta_{p q}$ equals $s\left(f_{j}\right)-1$, as a result, objective function coefficient of $\gamma_{j}$ equals 1 . For example, in the above instance, considering $f_{1}, T_{4}$ is located next to $T_{1}$, and $T_{1}$ is adjacent to $T_{3}$, thus $\sum_{T_{p} \& T_{q} \in f_{1} \mid p<q} \theta_{p q}=2$ and coefficient of $\gamma_{1}$ equals 1 . At the worst case, none of activities of $f_{j}$ is placed right next to another activity of $f_{j}$, consequently $\sum_{T_{p} \& T_{q} \in f_{j} \mid p<q} \theta_{p q}=0$, thus the objective function coefficient for $\gamma_{j}$ will be equal to zero. For example, considering the second factor in the above instance, $T_{2}$ is not located next to $T_{4}$, thus $\sum_{T_{p} \& T_{q} \in f_{2} \mid p<q} \theta_{p q}=0$ and thus coefficient of $\gamma_{2}$ equals 0 too. As another example, let's consider the third factor. Because $T_{4}$ and $T_{1}$ are adjoining, but $T_{2}$ is not located next to $T_{4}$ or $T_{1}$, this results in $\sum_{T_{p} \& T_{q} \in f_{3} \mid p<q} \theta_{p q}=1$, i.e. coefficient of $\gamma_{3}$ equals 0.5 .

Each activity might have at most two neighbourhoods in any solution. This limitation is observed by Inequalities (3). From the point of view of mathematical field of graph theory, these constraints cause the Model (2)-(5) generate only simple trees instead of unacceptable trees such as Figure 1-a.

The solution of the problem should be a contiguous chain of all the activities. As a result, no cycle is accepted. Constraints (4) prevent generating cyclic patterns such as Figure 1-b.

After having solved the Model (2)-(5), two issues should be handled. First, the solution may be nonconnected trees such as Figure 1-c in which we need to join them to make a linear arrangement of all activities. Of course, it is possible to add Constraint (6) to the Model (2)-(5) to prevent generation of non-connected trees. But, adding this constraint is not advised, because it causes the model randomly connects the parts of nonconnected tree. Instead, we advise the experts compare the tail-end activities of non-connected parts of trees, and then determine the most similar activities as joining points. As the second issue, we need to determine the direction of the arranged structure of activities from the most important to the least important. This aim also can be reached by comparing two tail-end activities of the structure using the expert's opinions.

$$
\sum_{p=1}^{m} \sum_{q=1}^{m} \theta_{p q}=m-1
$$

\section{$\underline{\text { Insert Figure } 1 \text { here }}$}




\section{Cases studied}

\subsection{Transformer oil commercialization}

The focus of this case concerns a project of the know-how commercialization of a technology known in the industry as transformer oil. This technology is a highly-refined mineral oil that is stable at high temperatures and has excellent electrical insulating properties. It is used in oil-filled transformers, some high voltage capacitors, fluorescent lamp ballasts, and some types of high voltage switches and circuit breakers. Transformer oil is used in oil-immersed transformers, high voltage capacitors, tap changers, fluorescent lamp ballasts, and some switches and circuit breakers.

In one of the project modules, it was needed to prioritize seven major independent activities as in Table 1. Two experts were involved in this case. The importance of the experts was identified as $u_{1}=1$ and $u_{2}=0.5$. The experts recommended five factors \#1 to \#5. The first expert proposed the factors weights 8, 8, 5, 4, and 7 respectively. The latter gave only ideas on the factors $\# 2$ and $\# 5$ with $w_{22}=3.5$ and $w_{52}=9$. The final weights of the factors were obtained as $\gamma_{1}=\left(8^{1}\right)^{1 / 1}=8, \gamma_{2}=\left(8^{1} \times 3.5^{0.5}\right)^{1 / 1.5}=6.07, \gamma_{3}=\left(5^{1}\right)^{1 / 1}=5$, $\gamma_{4}=\left(4^{1}\right)^{1 / 1}=4$, and $\gamma_{5}=\left(7^{1} \times 9^{0.5}\right)^{1 / 1.5}=7.61$. The model (2)-(5) was formulated with 21 variables and 105 constraints. This model was solved with the use of the LINGO release 8.0 on a personal computer. The optimal arrangement of the activities was obtained as $T_{7} T_{5} T_{4} T_{1} T_{2} T_{3} T_{6}$ corresponding to the objective value 26.68. Therefore, factor \#4 is the only one whose activities include a gap. The experts determined $T_{6}$ as the most important activity.

\section{$\underline{\text { Insert Table } 1 \text { here }}$}

\subsection{Planning at NIOC}

National Iranian Oil Company (NIOC), according to its mission on oil production from the country's reservoirs, compiles five-year productions and investment plans for all oil and gas fields in the country. This task is the responsibility of the NIOC's project planning management deputy. In this regard, this deputy receives final reports of several studies made by other deputies (such as deputy for hydrocarbon reservoirs, deputy for economic feasibility and investment, deputy for oil and gas projects, and so on), and analyses them to make investment plan.

The current case studied, reports a real project at the NIOC in which the output of eight studies was received to be analysed by the relevant team. Therefore, eight activities were defined as:

- $T_{1}$ : Analysing reservoir study.

- $T_{2}$ : Analysing surface and downstream study.

- $T_{3}$ : Analysing the study of target markets. 
- $T_{4}$ : Analysing the study of energy capital financing.

- $T_{5}$ : Analysing the study of the type of development and maintenance contracts.

- $T_{6}$ : Analysing of oil transfer and transportation study.

- $\quad T_{7}$ : Analysing the evaluation of similar projects.

- $T_{8}$ : Analysing the production of crude oil.

Due to the limited human resources in the team (i.e., technical and planning experts), the activities had to be done sequentially. Moreover, based on four factors, defined by the planning management of the NIOC, the following groups of activities were determined to be done continuously:

- Activities $T_{1}$ and $T_{2}$ (due to engineering and reservoir factor, with weight 100).

- Activities $T_{3}, T_{6}$, and $T_{8}$ (due to international market factor, with weight 40 ).

- Activities $T_{4}$ and $T_{5}$ (due to economic feasibility factor, with weight 70 ).

- Activities $T_{4}$ and $T_{7}$ (due to technology factor, with weight 40 ).

Using the proposed model, the optimal solution was obtained including three non-connected trees $T_{5} T_{4} T_{7}, T_{1} T_{2}$ and $T_{6} T_{3} T_{8}$. Finally, according to the expert's opinions, the linear arrangement of activities was determined as $T_{1} T_{2} T_{7} T_{4} T_{5} T_{6} T_{3} T_{8}$ with the best objective value 250 .

\section{Dealing with large scale problems}

The Model (2)-(5) includes $m^{2} / 2-m / 2$ binary variables and $2^{m}-m^{2} / 2+m / 2$ constraints $(m>2)$. Hence, the constraints exponentially grow in number by increasing $\mathrm{m}$. For example, there are 22 constraints for $m=5$, 979 constraints for $m=10$, and 32663 constraints for $m=15$. The problem was modelled as binary programming that is a subset of integer programming. Since integer programming is NP-hard [36, 37], the problem must also be hard. Due to the involved NP-hardness of the problem, heuristic and meta-heuristic approaches may strike us. Despite this, the current paper aims at looking for a method that provides global optimal solution. To solve large scale models, the paper suggests a relaxation that is removing Constraints (4) from the Model (2)-(5), resulting in a relaxed model with only $m$ constraints. Needless to say, the solution pattern of the relaxed model may include cycles and non-connected sub-graphs. Definitely, the output pattern may be one of two cases: a solution pattern i.e. a linear layout of all of activities, or a non-connected graph which includes at least one cycle. Accordingly, at first, we should identify any cycles, if those exist. With this purpose in mind, a pseudo code is designed as Figure 2. This code, in each round, arbitrarily chooses an initial vertex named "root", afterward, tries to search the next connected vertex to the initial vertex. The algorithm continues to search for the next vertex connected to the previous found one until getting the root or not finding the next connected vertex. The former shows a cycle and the latter indicates a chain. 


\section{Insert Figure 2 here}

Obviously, if there were no cycles (i.e., $t=0$ ), the current pattern is the optimal solution; otherwise, there would be one or more cycles. Therefore, in order to prevent generating the cycles, under a row generation approach, new Constraints (7) will be added to the Model (2)-(5), where $V_{r}$ is the set of vertices (i.e. activities) for the $r$ th cycle. This approach is often called branch-and-cut algorithm. Moreover, it should be noted that the row generation approach is a dual form of column generation approach [38].

$\sum_{T_{p} \& T_{q} \in V_{r} \mid p<q} \theta_{p q} \leq s\left(V_{r}\right)-1, \quad \forall r=1,2, \ldots, t$.

Let us, in summing up, review the proposed procedure. At the first iteration, the Model (2)-(5) is solved without Constraints (4) (i.e., $t=0$ ). Now, subject to the presence of any cycle in the generated pattern, the relevant Constraint (7) would be added into the model. Admittedly, this version of model includes Constraints (7) instead of Constraints (4). This model should be solved and rechecked for presence of cycles again. This iterative process continues until no cycle can be found.

\section{Experimental evaluation}

As a preliminary assumption, we assume that there are three parameters affecting running time: the number of activities $(m)$, the number of factors $(n)$, and the size of factors $(s)$. Consequently, a typical problem is addressed by $(m, n, s)$ i.e., a problem with $m$ activities, and $n$ factors by size $s$. By dint of the fact that the current study is the first research to consider the introduced problem, naturally there was no benchmark instance in the literature. Hence, the instance problems (i.e. data set) were randomly generated by a macro module program in Excel software. LINGO software version 8.0 was used to solve the mathematical models along with an Excel macro to identify cycles. The procedure was run on an ASUS Core (i7-7500) $2.7 \mathrm{GHz}$ with $12.00 \mathrm{~GB}$ memory.

\subsection{Sensitivity analysis}

For the purpose of sensitivity analysis, the study is conducted on several instance problems with the number of activities ranging from $m=25$ to $m=300$, the number of factors ranging from $n=100$ to $n=2500$, and the sizes of factors ranging from $s=3$ to $s=75$. Figures 3-a, 3-b, 3-c, 3-d, 3-e and 3-f portray running time plots resulting from solving instance problems. All reported times are average of ten times running the algorithm on each level of the parameters.

Figures 3-a, 3-b and 3-c show variation in running time with $s$, each curve under a fixed $m$ and $n$. These figures indicate that increasing $\mathbf{S}$ often results in greedy growing running time. Interestingly, we found out that for $n=500$ and $n=1000$ a peak is occurred for $s$ around 10. Figures 3-d and 3-e present how running time varies as $n$ is grown. These figures are made with fixed $m$ (i.e., $m=100$ and $m=150$ ), and three levels $s=10$, $s=20$, and $s=30$. The curves in Figure 3-d show a slow incremental effect of $n$ on the running time of 
instance problems even up to 2500 factors. On the contrary, Figure 3-e does not follow the same trends as curves in Figure 3 -d. The curves have increasing trend up to around $n=1550$, followed by an abrupt drop, such that there is a rather uniform trend after $n=1600$. Figure 3-b confirms this phenomenon, too; where the curve of $n=2000$ is very under the curve of $n=1500$. Figure 3 -f shows the variation in running time with $n$. This figure is drawn based on instance problems by $n=500$ and four levels of $s$. The results of analysis illustrated that the curves as in Figure 3-f are the only ones with exponential growth. Almost all the curves show exponential growth in running time, when $m$ increases. Except curve with $s=3$, on the other cases there were large scale instance problems (about $m=250$ to $m=300$ activities) with running time even more than 24 hours, only for the first iteration of the solving procedure.

\section{$\underline{\text { Insert Figure } 3 \text { here }}$}

Regarding unusual variations in Figures 3-c and 3-e, we could not find out a definite interpretation except that it may because of intrinsic connection among factors. It should be noted that all the solved instance problems obtained the optimal solution through between 3 to 12 iterations. Further, as the figures show, running times range between 1 and 3500 seconds. In sum, the running times are very reactive (i.e., alert) to $m$, and slightly sensitive to $S$ and $n$.

\subsection{Efficiency analysis}

For the sake of demonstrating the efficiency of the developed row generation algorithm, the employed approach would be as try to solve the test problems using the un-relaxed Model (2)-(5) as much as possible depending on running time or computer memory limitations, and then solve the same test problems using the row generation algorithm. This will show the effectiveness of the row generation algorithm over the un-relaxed Model (2)-(5). In order to determine the levels of parameters $(m, n$, and $s)$ to generate the instance problems, the major result of the previous section, sensitivity analysis, was used. Correspondingly, the number of activities $(\boldsymbol{m})$ is considered as the main parameter to be varied, with two levels of the other parameters as low $(n=m$ and $s \cong 0.25 m)$ and high $(n=3 m$ and $s \cong 0.75 m)$. Table 2 presents the characteristics and results of 10 test problems with the number of activities ranging from $m=5$ to $m=19$ for the un-relaxed Model (2)-(5) compared with the relaxed version. It was not possible to bring in the instances with more activities due to computer memory limitations (after verifying that using greater number of activities did not affect the obtained results). For comparison, the two last columns in Table 2 were considered. The former, decreasing in constraints, is defined as one minus the ratio of the total number of constraints in the un-relaxed Model (2)-(5) to that in the relaxed model. The latter, decreasing in running time, is similar to the former except that it concerns running time instead of the total number of constraints. Unquestionably, the results confirm the efficiency of the proposed algorithm.

Insert Table 2 here 


\subsection{Running time analysis}

This section answers this question: "What size of the problems can be solved with the use of the proposed branch-and-cut algorithm in a logical time?" Among many solved problems, the results for average running time of 45 typical tested problems are shown in Table 3, sorted by running time. Problems P43 to P45 are typical instances with very high running time. We can conclude that the proposed procedure is undeniably useful to get the optimal solution for the problems including even up to 250 or some more activities in at most 60 minutes. But, when the number of activities is going on around 300 activities or more, the running time may unexpectedly jump to even more than 24 hours.

\section{$\underline{\text { Insert Table } 3 \text { here }}$}

\section{Concluding remarks}

To start with, this paper defined a special problem of prioritizing activities (named the APP) in which the aim is to handle activities one after another, by a serial form. Later, the notion of making a solution for the problem with the use of a mathematical model was explained. Studying on the state-of-the art of optimization and decision analysis, it is found out that this idea has not been studied. Taking this fact into considerations, the first contribution of the research was in introducing the idea of prioritizing activities as an optimization problem. Additionally, the challenge of large scale problems was also discussed, and next, a branch-and-cut procedure to deal with such situations was developed. Thereby, another contribution of the research is in demonstrating how a row generation approach can be used to get the optimal solution of the APP. The evaluations and discussions presented the efficiency and usability of the methodology for large scale problems even for instances of up to 250 activities. In conclusion, the proposed methodology has a promising future and practical applications in project decision making challenges in a productive manner. Further researches can be undertaken in (I) applying the proposed method in complex and large size real-world cases, and (II) developing heuristic algorithms to solve the problem.

\section{Appendix}

In order to illustrate how the Formulation (2)-(5) is written, this appendix provides a numerical example for a small problem. This problem includes five activities $T_{1}$ to $T_{5}$, and six factors with relevant sets as $f_{1}=\left\{T_{3}, T_{4}\right\}$, $f_{2}=\left\{T_{1}, T_{3}\right\}, f_{3}=\left\{T_{1}, T_{5}\right\}, f_{4}=\left\{T_{1}, T_{2}\right\}, f_{5}=\left\{T_{1}, T_{3}, T_{4}, T_{5}\right\}$, and $f_{6}=\left\{T_{1}, T_{2}, T_{4}\right\}$. The weights of factors are $\gamma_{1}=9, \gamma_{2}=7, \gamma_{3}=8, \gamma_{4}=2, \gamma_{5}=7$, and $\gamma_{6}=3$. The mathematical Model (2)-(5) for this problem is:

$$
\begin{aligned}
& \operatorname{Max} 9\left(\theta_{34}\right)+7\left(\theta_{13}\right)+8\left(\theta_{15}\right)+2\left(\theta_{12}\right)+7\left(\frac{\theta_{13}+\theta_{14}+\theta_{15}+\theta_{34}+\theta_{35}+\theta_{45}}{3}\right)+3\left(\frac{\theta_{12}+\theta_{14}+\theta_{24}}{2}\right) \\
& \theta_{12}+\theta_{13}+\theta_{14}+\theta_{15} \leq 2 \\
& \theta_{12}+\theta_{23}+\theta_{24}+\theta_{25} \leq 2
\end{aligned}
$$


$\theta_{13}+\theta_{23}+\theta_{34}+\theta_{35} \leq 2$

$\theta_{14}+\theta_{24}+\theta_{34}+\theta_{45} \leq 2$

$\theta_{15}+\theta_{25}+\theta_{35}+\theta_{45} \leq 2$

$\theta_{12}+\theta_{13}+\theta_{23} \leq 2$

$\theta_{12}+\theta_{14}+\theta_{24} \leq 2$

$\theta_{12}+\theta_{15}+\theta_{25} \leq 2$

$\theta_{13}+\theta_{14}+\theta_{34} \leq 2$

$\theta_{13}+\theta_{15}+\theta_{35} \leq 2$

$\theta_{14}+\theta_{15}+\theta_{45} \leq 2$

$\theta_{23}+\theta_{24}+\theta_{34} \leq 2$

$\theta_{23}+\theta_{25}+\theta_{35} \leq 2$

$\theta_{24}+\theta_{25}+\theta_{45} \leq 2$

$\theta_{34}+\theta_{35}+\theta_{45} \leq 2$

$\theta_{12}+\theta_{13}+\theta_{14}+\theta_{23}+\theta_{24}+\theta_{34} \leq 3$

$\theta_{12}+\theta_{13}+\theta_{15}+\theta_{23}+\theta_{25}+\theta_{35} \leq 3$

$\theta_{12}+\theta_{14}+\theta_{15}+\theta_{24}+\theta_{25}+\theta_{45} \leq 3$

$\theta_{13}+\theta_{14}+\theta_{15}+\theta_{34}+\theta_{35}+\theta_{45} \leq 3$

$\theta_{23}+\theta_{24}+\theta_{25}+\theta_{34}+\theta_{35}+\theta_{45} \leq 3$

$\theta_{12}+\theta_{13}+\theta_{14}+\theta_{15}+\theta_{23}+\theta_{24}+\theta_{25}+\theta_{34}+\theta_{35}+\theta_{45} \leq 4$

$\theta_{12}, \theta_{13}, \theta_{14}, \theta_{15}, \theta_{23}, \theta_{24}, \theta_{25}, \theta_{34}, \theta_{35}, \theta_{45} \in(0,1)$

To this example, the LINGO code and the solution are presented as Figure 4, which result in the optimal string as $T_{5} T_{1} T_{3} T_{4} T_{2}$. This corresponds to the objective value 32.5 .

Insert Figure 4 here 


\section{References}

1. Gerardi, B. "No-Drama Project Management: Avoiding Predictable Problems for Project Success", 1st Edn., pp. 2-3, Apress, NY, USA (2011).

2. Birjandi, A., Mousavi, S.M. and Vahdani, B. "Designing a resource-constrained project scheduling model considering multiple routes for flexible project activities: Meta-heuristic algorithms”, Scientia Iranica E, 27(5), pp. 2572-2591 (2020).

3. Namazian, A., Haji Yakhchali, S. and Rabbani, M. "Integrated bi-objective project selection and scheduling using Bayesian networks: A risk-based approach”, Scientia Iranica E, 26(6), pp. 3695-3711 (2019).

4. Dorfeshan, Y., Mousavi, S.M., Vahdani, B., et al. "Determining project characteristics and critical path by a new approach based on modified NWRT method and risk assessment under an interval type-2 fuzzy environment", Scientia Iranica E, 26(4), pp. 2579-2600 (2019).

5. Pheng, L.S. and Chuan, Q.T. "Environmental factors and work performance of project managers in the construction industry", Int. J. Project Management, 24, pp. 24-37 (2006).

6. Turner, J.R. and Muller, R. "The project manager's leadership style as a success factor on projects: a literature review", Project Management J., 36(1), pp. 49-61 (2005).

7. Berjis, N., Shirouyehzad, H. and Jouzdani, J. "A new approach to determine the weights of project activities using data envelopment analysis: a case of Mobarakeh steel company”, Int. J. Managing Projects in Business, 13 (6), pp. 1187-1217 (2020).

8. Greek, D. and Pullin, J. “Overrun, overspent, overlooked”, Professional Engineering, 12(3), pp. 27-8 (1999).

9. Chang, T.C. and Ibbs, C.W. "Priority ranking: A fuzzy expert system for priority decision making in building construction resource scheduling”, Building and Environment, 25, pp. 253-267 (1990).

10. Babauta, L. "Zen To Done: The Ultimate Simple Productivity System”, 1st Edn., LLC, Phatbits, UK (2012).

11. Covey, S.R. "7 Habits of Highly Effective People", 25th Edn., Pocket books, UK (1999).

12. Baykasoglu, A., Gocken, T. and Kaplanoglu, V. "A practical approach to prioritize project activities through fuzzy ranking", Cybernetics and Systems: An International Journal, 42(3), pp. 165-179 (2011).

13. Alencar, L.H., Mota, C.M.M., and Almeida, A.T. "Activities prioritization in a construction project management based on multicriteria methods", Third International Conference on Production Research, Americas' Region, Federac a a o da Indu' strias do Estado do Parana', Brazil, July 30-August 2 (2006).

14. Jha, K.N. and Misra, S. "Ranking and classification of construction coordination activities in Indian projects", Construction Management and Economics, 25, pp. 409-421 (2007).

15. Mota, C.M.M., Almeida, A.T. and Alencar, L.H. "A multiple criteria decision model for assigning priorities to activities in project management", Int. J. Project Management, 27, pp. 175-181 (2009).

16. Vanhoucke, M. "Using activity sensitivity and network topology information to monitor project time performance", Omega, 38(5), pp. 359-370 (2010).

17. Mota, C.M.M. and de Almeida, A.T. "A multi-criteria decision model for assigning priority classes to activities in project management”, Annals of Operations Research, 199(1), pp. 361-372 (2012).

18. Golpira, H. "Making comparison between FMCDM and MCDM to define project activities weights", First International Scientific Conference on Project Management in the Baltic Countries, February 8-9, Riga, University of Latvia, Vol. 35 (2012).

19. Hadad, Y. Keren, B. and Laslo, Z. "Applications of ranking indexes of project activities for project management tasks", Challenges of IT Project Porffolio Selection, 1392, pp. 1422 (2014).

20. Hadad, Y. "Multi-criteria methods for ranking project activities”, Yugoslav J. Operations Research, 26(2), pp. 201-219 (2016).

21. Kalayathankal, S.J., Varghese, J. and Abraham, J.T. “A modified fuzzy approach to prioritize project activities”, Int. J. Engineering \& Technology, 7(2), pp. 158-162 (2018).

22. Hwang, C.L. and Yoon, K. "Multiple Attribute Decision Making: Methods and Applications: A State-of-the-art Survey", 1st Edn., Springer, Berlin (1981).

23. Zavadskas, E.K. and Podvezko, V. "Integrated determination of objective criteria weights in MCDM", Int. J. Information Technology \& Decision Making, 15(2), 267-283 (2016). 
24. Vinogradova, I., Podvezko, V. and Zavadskas, E.K. "The recalculation of the weights of criteria in MCDM methods using the Bayes approach”, Symmetry, 10(205), pp. 2-18 (2018).

25. Hatefi, M.A. “A model for measuring success of an organisational management system”, Int. J. Advanced Operations Management, 9(2), pp. 73-87 (2017).

26. Pinheiro, R.G.S., Martins, I.C., Protti, F., et al. "A matheuristic for the cell formation problem”, Optimization Letters, 12(2), pp. 335-346 (2018)

27. Rabbani, M., Habibnejad-Ledari, H., Rafiei, H., et al. "A bi-objective mathematical model for dynamic cell formation problem considering learning eect, human issues, and worker assignment", Scientia Iranica E, 23(5), pp. 2341-2354 (2016).

28. Leusin, M.E., Frazzon, E.M., Maldonado, M.U., et al. "Solving the Job-Shop Scheduling Problem in the Industry 4.0 Era”, Technologies, 6(107), pp. 2-27 (2018).

29. Palubeckis, G. "Single row facility layout using multi-start simulated annealing", Computers \& Industrial Engineering, 103, pp. 1-16 (2017).

30. Ceberio, J., Mendiburu, A. and Lozano, J.A. “The linear ordering problem revisited”, Eur. J. Operational Research, 241(3), pp. 686696 (2015).

31. Horton, S.B., Gary Parker, R. and Borie, R.B. "On minimum cuts and the linear arrangement problem", Discrete Applied Mathematics, 103, pp. 127-139 (2000).

32. Glover, F. and Rego, C. "New relationships for multi-neighbourhood search for the minimum linear arrangement problem", $J$. Discrete Algorithms, 46-47, pp. 16-24 (2017).

33. Seppanen, J. and Moore, J.M. "Facilities planning and graph theory", Int. J. Management Science, 17, pp. 242-253 (1970).

34. Hatefi, M.A. "BRAW: block-wise rating the attribute weights in MADM", Computers \& Industrial Engineering, 156, 107274 (2021).

35. Hillier, F. and Lieberman, G. "Introduction to Operations Researcb”, 11th Edn., McGraw-Hill Education, NY, USA (2020).

36. Garey, M.R. and Johnson, D.S. "Computers and intractability: A guide to the theory of NP completeness", WH Freeman, NY, USA, (1979).

37. Page, D.R., “Approximation algorithms for problems in make-span minimization on unrelated parallel machines”, Ph.D. Thesis, University of Western Ontario (2019).

38. Hatefi, M.A. "Developing column generation approach to solve the rectangular two-dimensional single knapsack problem", Scientia Iranica E, 24(6), pp. 3287-3296 (2017).

Dr. Mohammad Ali Hatefi is an associate professor of department of energy economics \& management at Petroleum University of Technology (PUT). He was graduated from Iran University of Science and Technology (IUST) with honor. His areas of interest are operations research, decision analysis, project management and risk management. He is the author of several scientific publications in the area of decision analysis, operations research and risk management. He is currently serving as chief of faculty of Tehran, a branch of PUT.

Dr. Seyyed Abdollah Razavi is an assistant professor of department of energy economics \& management at PUT. He was graduated from Ferdowsi University Mashhad in the field of energy economics. His areas of interests and researches are energy management, energy economics, financial market, and political economy. He is presently working for journal of Petroleum Business Review (PBR) as executive manager. 


\section{List of figure captions}

Figure 1. Infeasible patterns containing: (a) unacceptable tree, (b) cycle, and (c) non-connected tree

Figure 2. The pseudo code for cycle identification

Figure 3. Comparative analysis of running times (in seconds)

Figure 4. The LINGO code and the related solution report for the numerical example

\section{List of table captions}

Table 1. The activity-factor matrix for the case studied

Table 2. The characteristics and results of 10 instances for both the un-relaxed and relaxed forms

Table 3. The running time (in seconds) of the selected instances 


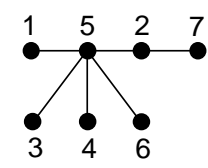

(a)

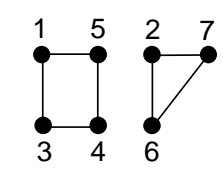

(b)

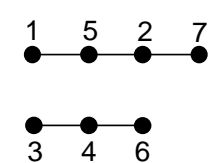

(c)

Figure 1. Infeasible patterns containing: (a) unacceptable tree, (b) cycle, and (c) non-connected tree 


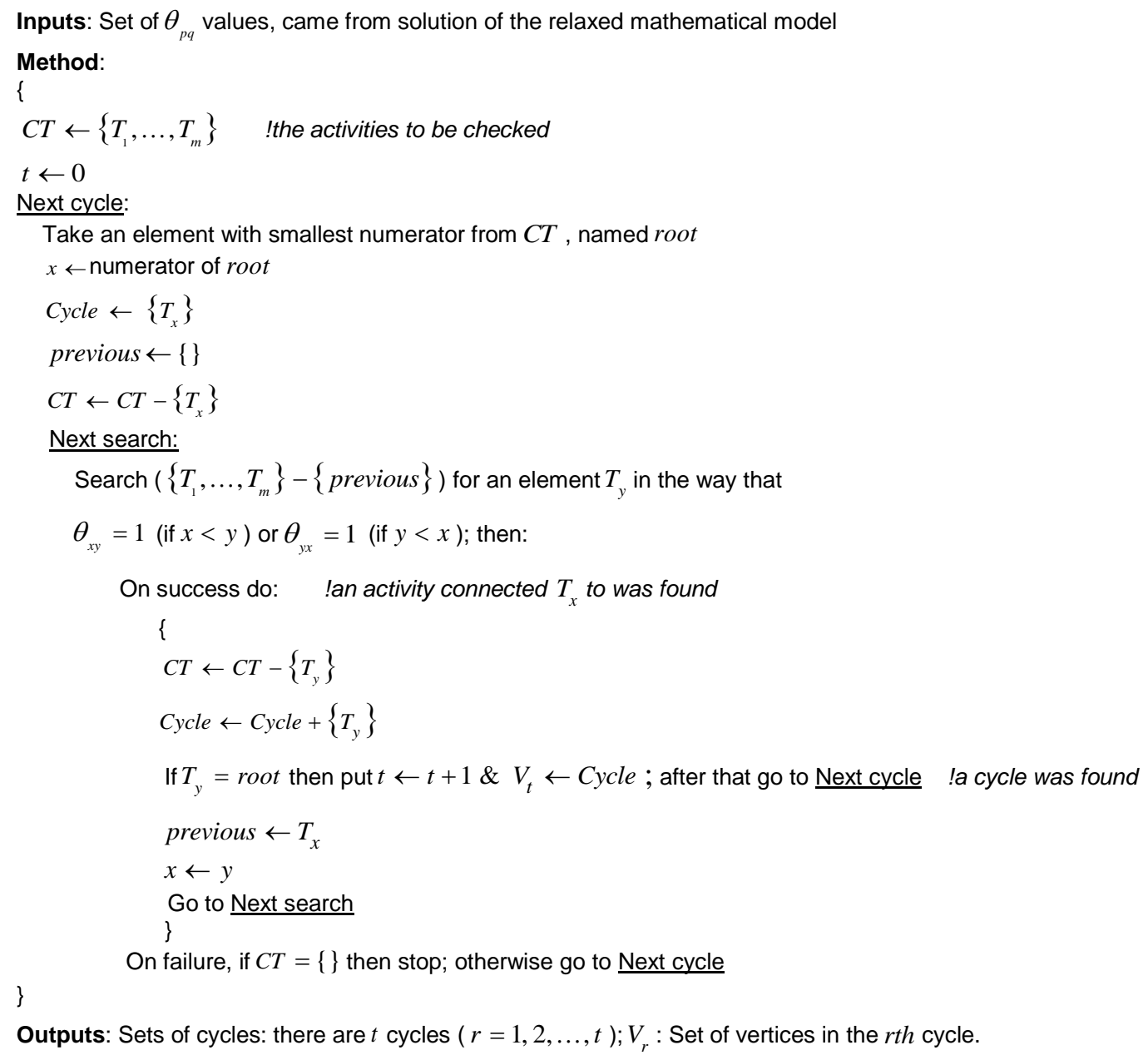

Outputs: Sets of cycles: there are $t$ cycles $(r=1,2, \ldots, t) ; V_{r}$ : Set of vertices in the $r$ th cycle.

Figure 2. The pseudo code for cycle identification 


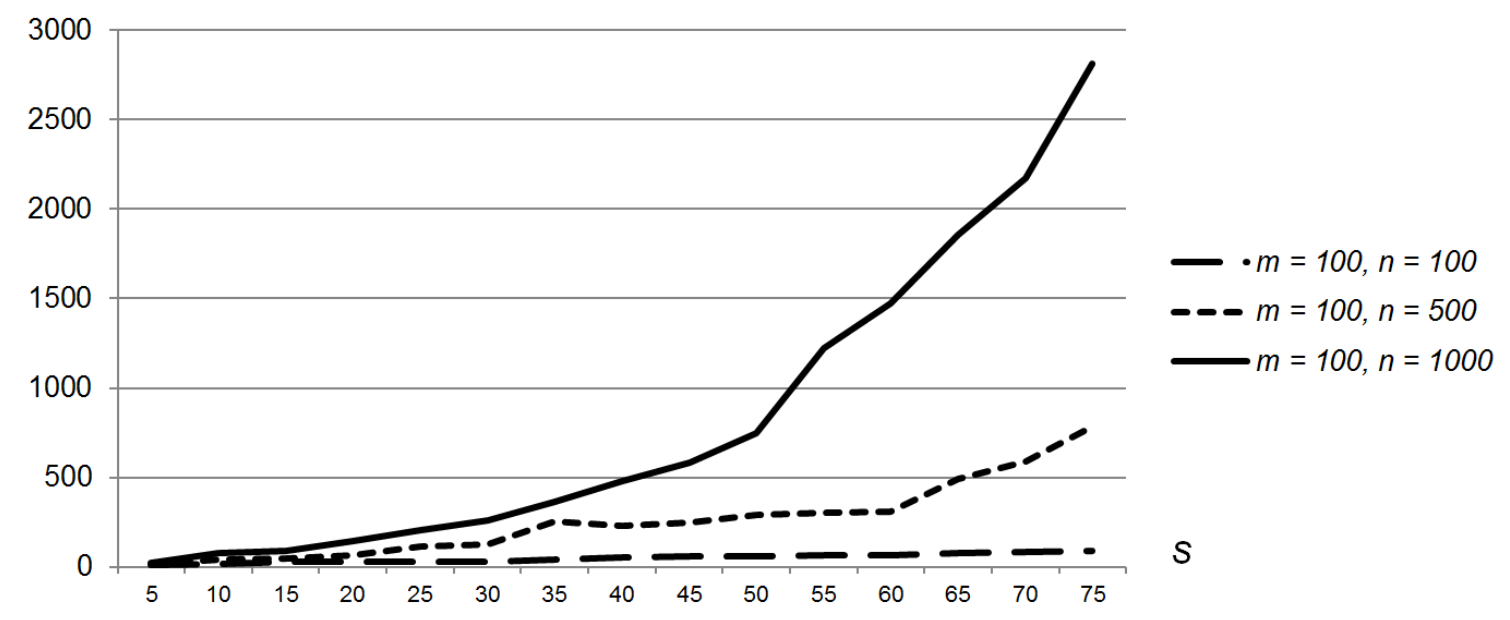

(a)

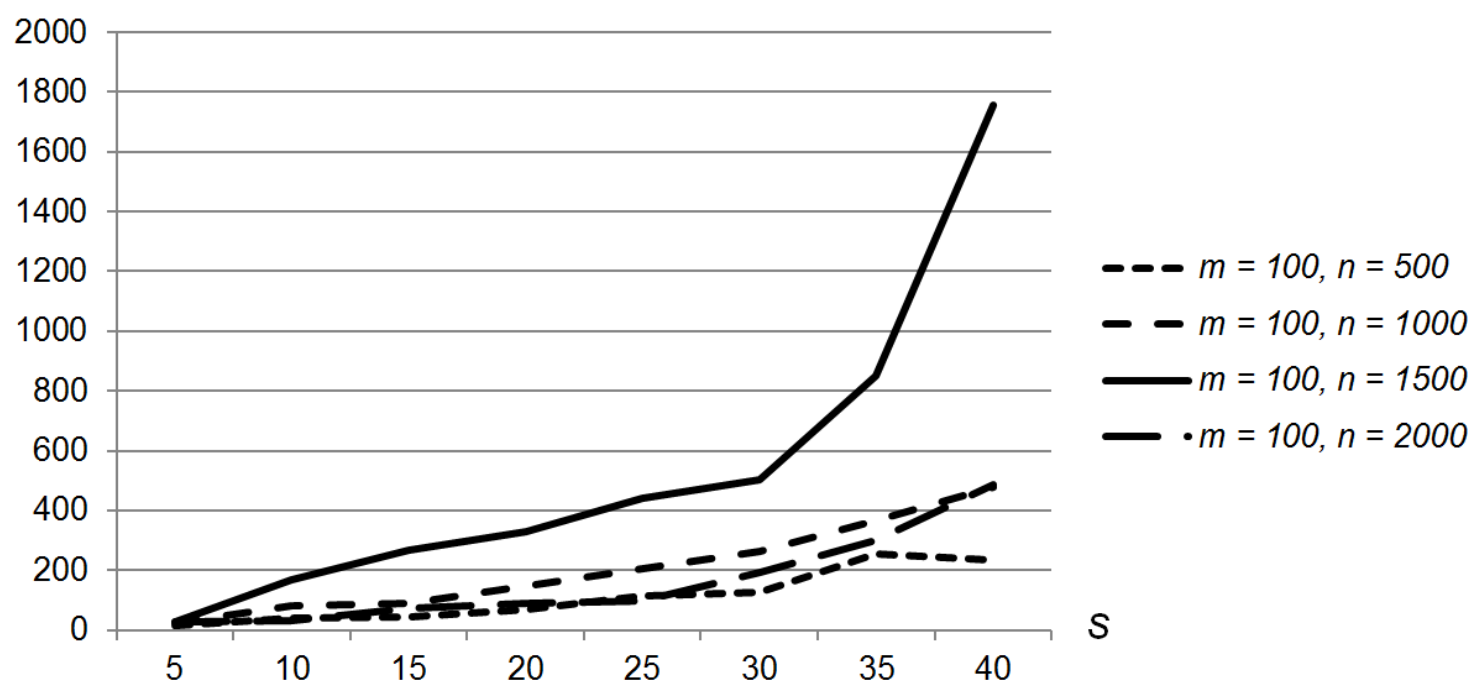

(b) 


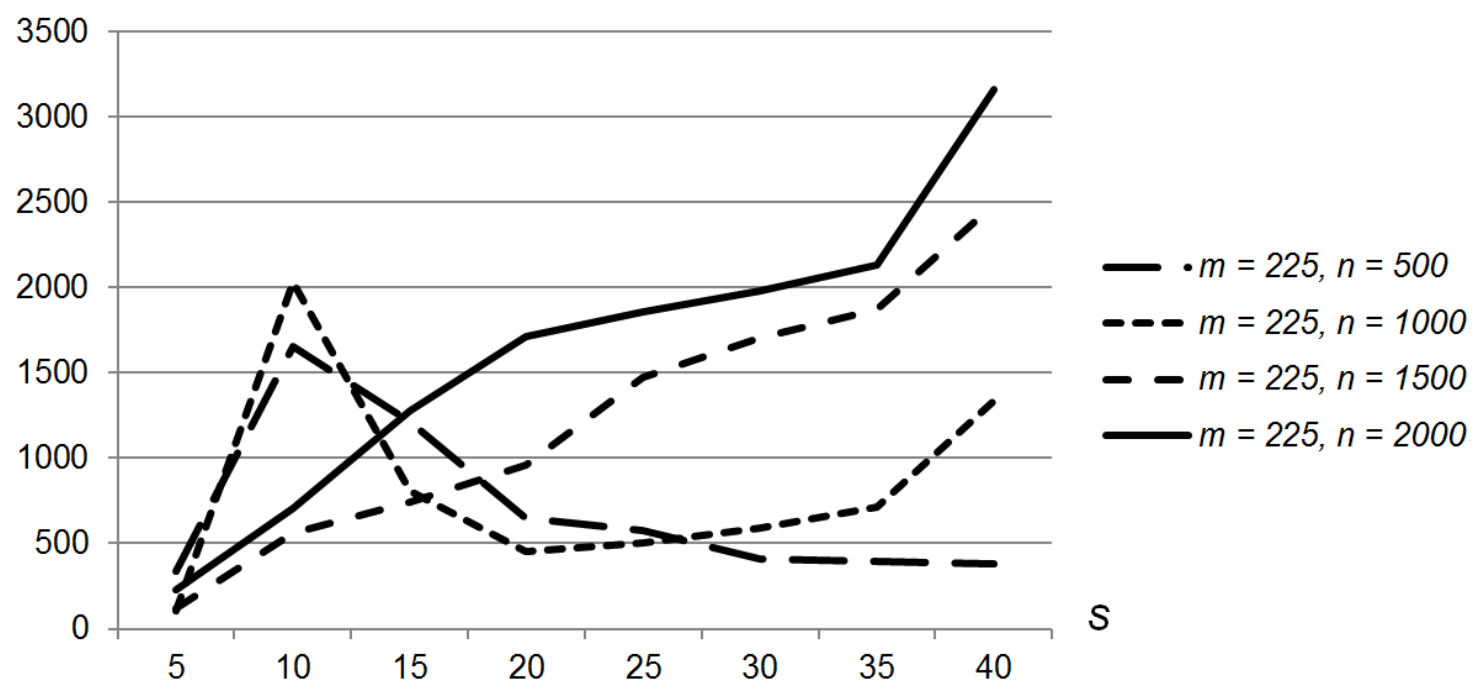

(c)

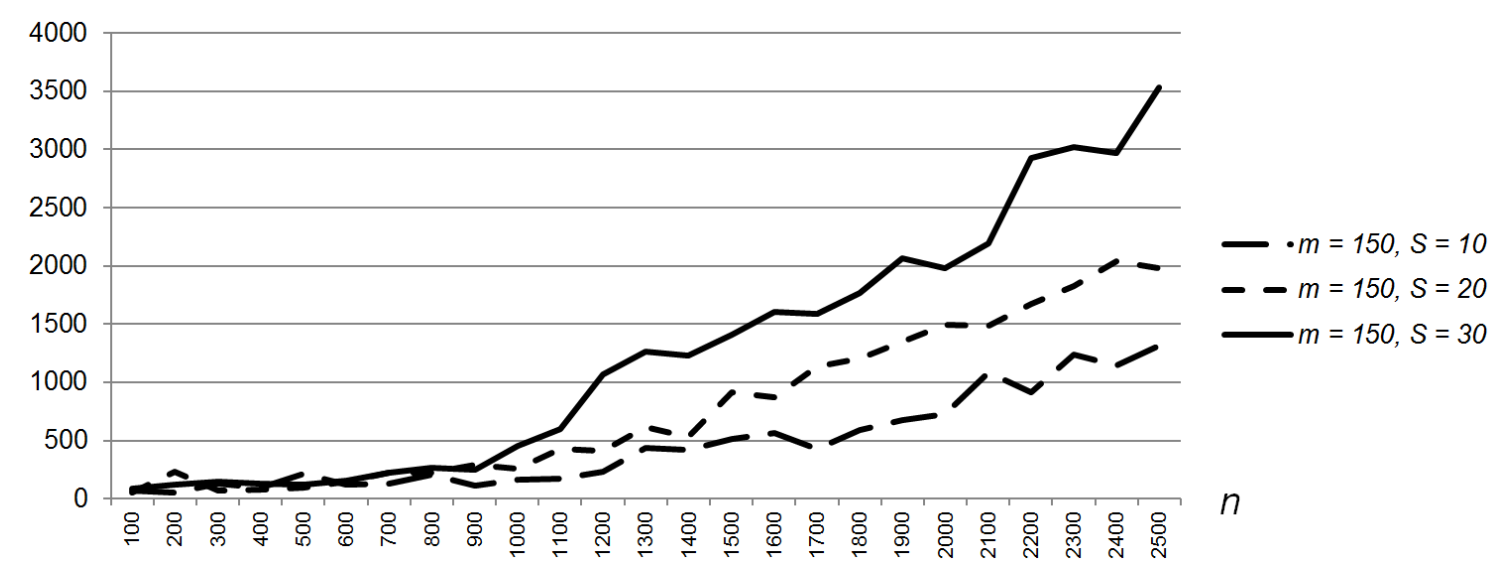

(d)

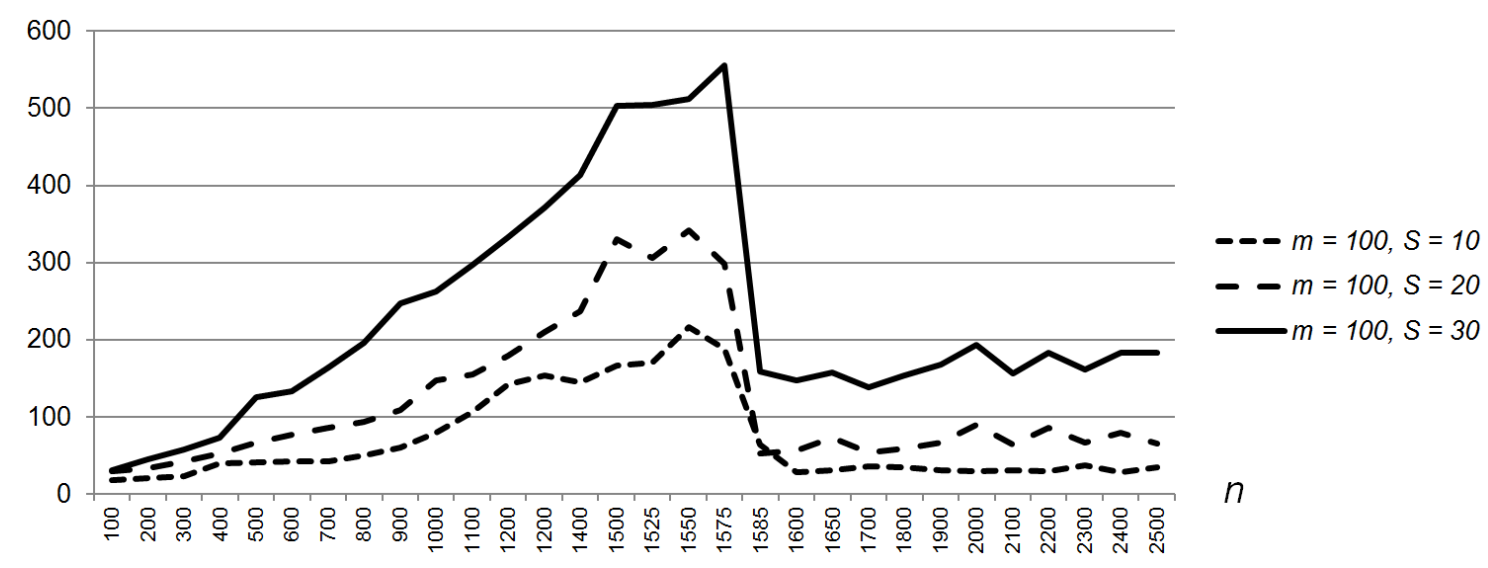

(e) 


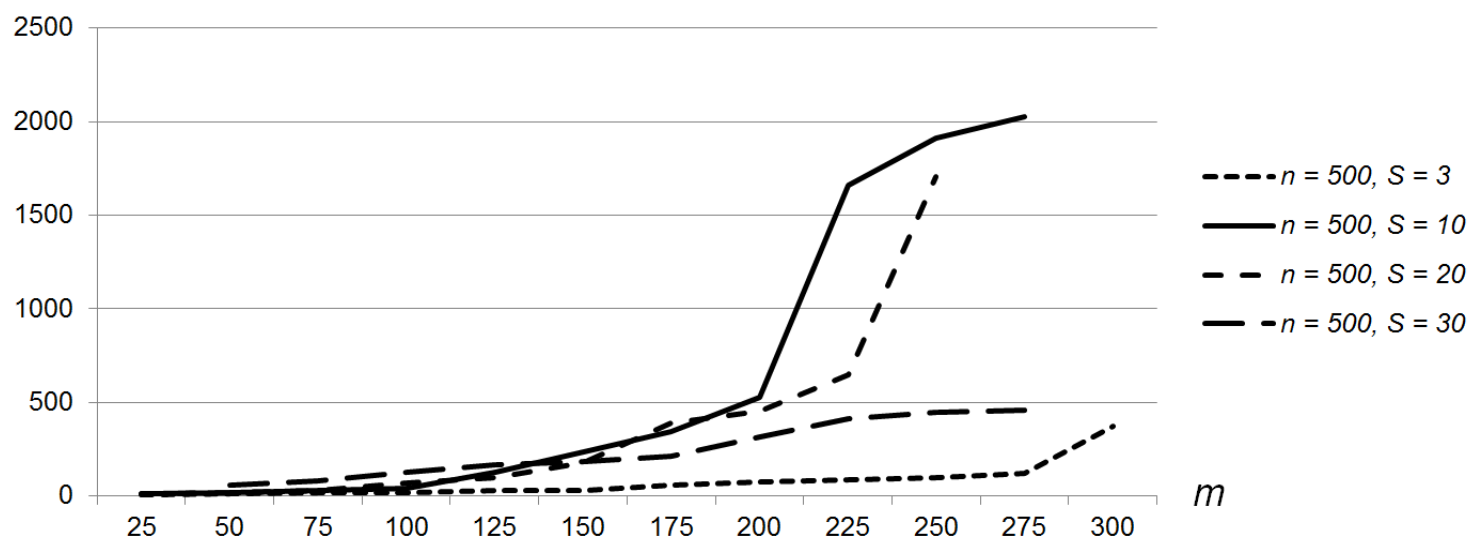

(f)

Figure 3. Comparative analysis of running times (in seconds)

Sets:

Activity/1..5/;

Pair (Activity, Activity)|\&2 \#GT\# \&1:Teta;

Endsets

$\operatorname{Max}=9^{\star} \operatorname{Teta}(3,4)+7^{*} \operatorname{Teta}(1,3)+8^{*} \operatorname{Teta}(1,5)+2^{\star} \operatorname{Teta}(1,2)+(7 / 3)^{\star}(\operatorname{Teta}(1,3)+\operatorname{Teta}(1,4)+\operatorname{Teta}(1,5)+\operatorname{Teta}(3,4)+\operatorname{Teta}(3,5)+\operatorname{Teta}(4,5))+$ $(3 / 2)^{\star}(\operatorname{Teta}(1,2)+\operatorname{Teta}(1,4)+\operatorname{Teta}(2,4))$;

@for(Activity(i): @sum(Pair(p,q)|p \#EQ\# i \#OR\# q \#EQ\# i: Teta(p,q))<=2);

@for(Activity(i1): @for(Activity(i2) | i2 \#GT\# i1: @for(Activity(i3) | i3 \#GT\# i2: +Teta(i1, i2)+Teta(i1, i3)+Teta(i2, i3)<= 2;)));

@for(Activity(i1): @for(Activity(i2) | i2 \#GT\# i1: @for(Activity(i3) | i3 \#GT\# i2: @for(Activity(i4) | i4 \#GT\# i3:

Teta(i1, i2)+Teta(i1, i3)+Teta(i1, i4)+Teta(i2, i3)+Teta(i2, i4)+Teta(i3, i4)<= 3;))));

$\operatorname{Teta}(1,2)+\operatorname{Teta} \square(1,3) \square+\operatorname{Teta}(1,4)+\operatorname{Teta}(1,5)+\operatorname{Teta}(2,3)+\operatorname{Teta}(2,4)+\operatorname{Teta}(2,5)+\operatorname{Teta}(3,4)+\operatorname{Teta}(3,5)+\operatorname{Teta}(4,5)<=4$;

@for(Pair(p,q): @Bin(Teta));

\begin{tabular}{|c|c|c|c|}
\hline Solution Report - Lingo sample & & $\square$ & $x$ \\
\hline Global optimal solution f & nd at iteratic & ר: $\quad 4$ & \\
\hline Objective value: & & 2.50000 & \\
\hline Variable & Value & Reduced Cost & \\
\hline $\operatorname{Teta}(1,2)$ & 0.000000 & -3.500000 & \\
\hline $\operatorname{Teta}(1,3)$ & 1.000000 & -9.333333 & \\
\hline Teta $(1,4)$ & 0.000000 & -3.833333 & \\
\hline Teta $(1,5)$ & 1.000000 & -10.33333 & \\
\hline Teta $(2,3)$ & 0.000000 & 0.000000 & \\
\hline Teta $(2,4)$ & 1.000000 & -1.500000 & \\
\hline Teta $(2,5)$ & 0.000000 & 0.000000 & \\
\hline $\operatorname{Teta}(3,4)$ & 1.000000 & -11.33333 & \\
\hline Teta $(3,5)$ & 0.000000 & -2.333333 & \\
\hline Teta $(4,5)$ & 0.000000 & -2.333333 & \\
\hline
\end{tabular}


Figure 4. The LINGO code and the related solution report for the numerical example

Table 1. The activity-factor matrix for the case studied

\begin{tabular}{lllllll}
\hline & \multicolumn{1}{c}{ Activities } & \multicolumn{5}{c}{ Factors } \\
\cline { 3 - 6 } & & 1 & 2 & 3 & 4 & 5 \\
\hline $\mathbf{T}_{\mathbf{1}}$ & Specifying electrically insulate requirements. & $*$ & $*$ & & & \\
$\mathbf{T}_{\mathbf{2}}$ & Specifying suppress corona and arcing equipment. & & $*$ & $*$ & $*$ & \\
$\mathbf{T}_{\mathbf{3}}$ & Studying on coolant features. & & $*$ & $*$ & & $*$ \\
$\mathbf{T}_{\mathbf{4}}$ & Determining resist oxidation items. & $*$ & $*$ & & & \\
$\mathbf{T}_{\mathbf{5}}$ & Determining deposit formation items. & $*$ & & & & \\
$\mathbf{T}_{\mathbf{6}}$ & Studying on the overall system performance. & & $*$ & & $*$ & $*$ \\
$\mathbf{T}_{\mathbf{7}}$ & Making the manual for installation of the system. & $*$ & & & & \\
\hline
\end{tabular}


Table 2. The characteristics and results of 10 instances for both the un-relaxed and relaxed forms

\begin{tabular}{|c|c|c|c|c|c|c|c|c|c|c|c|c|}
\hline \multirow[b]{2}{*}{$\begin{array}{l}\text { E } \\
\frac{0}{0} \\
0 \\
0\end{array}$} & \multirow[b]{2}{*}{$(m, n, s)$} & \multicolumn{4}{|c|}{ The un-relaxed form } & \multicolumn{5}{|c|}{ The relaxed form } & \multicolumn{2}{|c|}{ Comparison } \\
\hline & & $\begin{array}{l}\frac{\ddot{d}}{2} \\
\frac{\pi}{\pi} \\
\frac{\pi}{7}\end{array}$ & 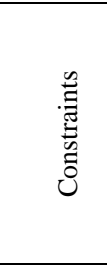 & 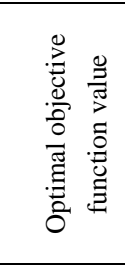 & 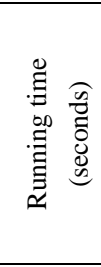 & 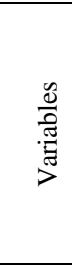 & 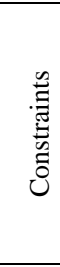 & 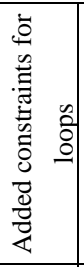 & 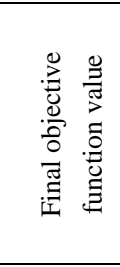 & 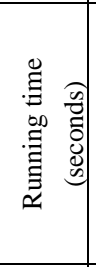 & 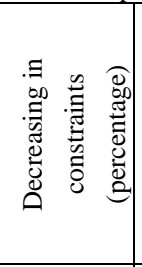 & 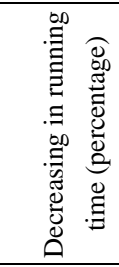 \\
\hline P1 & $(5,5,3)$ & 10 & 22 & 237.5 & 0.06 & 10 & 5 & 1 & 237.5 & 0.05 & $72.727 \%$ & $16.667 \%$ \\
\hline $\mathrm{P} 2$ & $(5,15,4)$ & 10 & 22 & 692.66 & 0.07 & 10 & 5 & 3 & 692.66 & 0.05 & $63.636 \%$ & $28.571 \%$ \\
\hline $\mathrm{P} 3$ & $(10,10,3)$ & 45 & 979 & 355 & 0.1 & 45 & 10 & 0 & 355 & 0.05 & $98.979 \%$ & $50.000 \%$ \\
\hline $\mathrm{P} 4$ & $(10,30,7)$ & 45 & 979 & 1319.5 & 0.1 & 45 & 10 & 1 & 1319.5 & 0.08 & $98.876 \%$ & $20.000 \%$ \\
\hline P5 & $(15,15,4)$ & 105 & 32663 & 536.66 & 3.32 & 105 & 15 & 4 & 536.66 & 0.07 & $99.942 \%$ & $97.892 \%$ \\
\hline P6 & $(15,45,11)$ & 105 & 32663 & 1949.5 & 6.04 & 105 & 15 & 3 & 1949.5 & 0.07 & $99.945 \%$ & $98.841 \%$ \\
\hline P7 & $(17,17,4)$ & 136 & 130936 & 467.33 & 66.13 & 136 & 17 & 9 & 467.33 & 0.1 & $99.980 \%$ & $99.849 \%$ \\
\hline P8 & $(17,51,13)$ & 136 & 130936 & 2290.583 & 23.9 & 136 & 17 & 4 & 2290.583 & 0.09 & $99.984 \%$ & $99.623 \%$ \\
\hline $\mathrm{P9}$ & $(19,19,5)$ & 171 & 524117 & 627 & 121.3 & 171 & 19 & 2 & 627 & 0.08 & $99.996 \%$ & $99.934 \%$ \\
\hline P10 & $(19,57,14)$ & 171 & 524117 & 2259 & 693 & 171 & 19 & 4 & 2259 & 0.12 & $99.996 \%$ & $99.983 \%$ \\
\hline
\end{tabular}


Table 3. The running time (in seconds) of the selected instances

\begin{tabular}{|c|c|c|c|c|}
\hline Problem & $m$ & $(m, n, s)$ & Running time & Average \\
\hline P1 & \multirow{13}{*}{150} & $(150,100,30)$ & 98 & \multirow{13}{*}{946.61} \\
\hline P2 & & $(150,500,30)$ & 126 & \\
\hline P3 & & $(150,500,10)$ & 567 & \\
\hline P4 & & $(150,1800,10)$ & 651 & \\
\hline P5 & & $(150,1900,10)$ & 798 & \\
\hline P6 & & $(150,200,20)$ & 896 & \\
\hline P7 & & $(150,2500,10)$ & 917 & \\
\hline P8 & & $(150,2000,20)$ & 1064 & \\
\hline P9 & & $(150,2200,10)$ & 1099 & \\
\hline P10 & & $(150,1500,30)$ & 1134 & \\
\hline P11 & & $(150,2100,10)$ & 1295 & \\
\hline P12 & & $(150,2500,10)$ & 1526 & \\
\hline P13 & & $(150,2000,30)$ & 2135 & \\
\hline P14 & \multirow{7}{*}{200} & $(200,500,10)$ & 105 & \multirow{7}{*}{443} \\
\hline P15 & & $(200,500,3)$ & 112 & \\
\hline P16 & & $(200,500,20)$ & 280 & \\
\hline P17 & & $(200,500,30)$ & 294 & \\
\hline P18 & & $(200,500,30)$ & 427 & \\
\hline P19 & & $(200,500,20)$ & 623 & \\
\hline P20 & & $(200,500,10)$ & 1260 & \\
\hline P21 & \multirow{3}{*}{225} & $(225,1500,5)$ & 91 & \multirow{3}{*}{1726} \\
\hline P22 & & $(225,500,5)$ & 189 & \\
\hline P23 & & $(225,2000,5)$ & 217 & \\
\hline
\end{tabular}




\begin{tabular}{|c|c|c|c|c|}
\hline Problem & $m$ & $(m, n, s)$ & Running time & Average \\
\hline P24 & & $(225,1000,10)$ & 266 & \\
\hline P25 & & $(225,2000,5)$ & 322 & \\
\hline P26 & & $(225,500,40)$ & 378 & \\
\hline P27 & & $(225,1000,20)$ & 476 & \\
\hline P28 & & $(225,1000,40)$ & 910 & \\
\hline P29 & & $(225,1500,20)$ & 959 & \\
\hline P30 & & $(225,2000,20)$ & 1715 & \\
\hline P31 & & $(225,500,5)$ & 2065 & \\
\hline P32 & & $(225,2000,30)$ & 2163 & \\
\hline P33 & & $(225,1000,10)$ & 6636 & \\
\hline P34 & & $(225,1000,10)$ & 7777 & \\
\hline P35 & \multirow{6}{*}{250} & $(250,500,3)$ & 105 & \multirow{6}{*}{1300.83} \\
\hline P36 & & $(250,500,20)$ & 252 & \\
\hline P37 & & $(250,500,10)$ & 693 & \\
\hline P38 & & $(250,500,20)$ & 1267 & \\
\hline P39 & & $(250,500,10)$ & 2492 & \\
\hline P40 & & $(250,500,20)$ & 2996 & \\
\hline P41 & \multirow{5}{*}{300} & $(300,500,3)$ & 413 & \multirow{5}{*}{-} \\
\hline P42 & & $(300,500,30)$ & 651 & \\
\hline P43 & & $(300,500,10)$ & More than 24 hours & \\
\hline P44 & & $(300,500,20)$ & More than 24 hours & \\
\hline $\mathrm{P} 45$ & & $(300,500,30)$ & More than 24 hours & \\
\hline
\end{tabular}

